\title{
Mitigating Cognitive Bottlenecks via an Augmented Cognition Adaptive System*
}

\author{
Michael C. Dorneich, Stephen D. Whitlow, Patricia May Ververs, William H. Rogers \\ Human Centered Systems COE \\ Honeywell Laboratories \\ Minneapolis, MN USA \\ michael.dorneich@honeywell.com
}

\begin{abstract}
A conceptual framework for designing humancomputer cognitive systems is proposed. The Cognitive Bottleneck Framework (CBF) identifies four significant "cognitive bottlenecks" that negatively impact the quality and tempo of decision making: (1) Information overload; humans cannot manage the vast amounts of information delivered by their computing environment, (2) Sequential cognitive processing; while information arrives in parallel, humans are essentially serial processors that can only address a single thread or task at a time, (3) Narrow user input capabilities: the system has more sophisticated means of communicating to the human than the human has of communicating to the system, and (4) Function mis-allocation; tasks are allocated to humans by default rather than by design, leaving them with tasks for which they are cognitively ill-suited. The overall purpose of CBF is to "right-size" these bottlenecks, remove constraints that restrict information flow, and better fit information channels to the abilities of either the human or computer.
\end{abstract}

Keywords: cognitive bottleneck, information overload, function allocation.

\section{Introduction}

One would think that the raw computational power of modern computers would reduce the mental workload of the users of automated systems. Certainly users no longer have to exert as much mental effort in computationally intensive tasks. However, and perhaps counter-intuitively, there are at least three pervasive, real-world drivers across domains and applications that actually spiral the mental workload of automation users upward: (1) the explosion of sensor and information processing technology provide never-seen-before quantities of information to users, but with little or no help in integrating it, and extracting its meaning or its implications for actions and decisions; (2) the never-ending drive for productivity-utopia - the incessant financial- and efficiency-driven desire to produce more work with fewer people; and (3) the culturally-driven pressure in civilized societies for flawless execution, even in probabilistic endeavors such as prediction, strategic planning, and decision making.

This all points to a common and increasing dilemma in high stakes, high information, high technology, ultradynamic environments where ultimate decision making and task execution still are human responsibilities: a heightened demand on the human cognitive system to achieve greater information processing throughput and accuracy, resulting in better and more rapid situation assessment, decision making, and execution of actions. Nowhere is this more obvious than on the modern military battlefield. There is no question that modern day military superiority is as much a function of information superiority as weapon, vehicle, or troop superiority. At the smallest units of action, even at the individual soldier level, humans are expected to process more information than ever, be aware of more aspects of the situation than ever, and make decisions faster and more accurately than ever. Users in other domains are no different; be it a control room, a classroom, or a board room, the back office, the front office, or the virtual office, the stock market, the real estate market, or the farmer's market; the need for faster, better, more accurate human information processing and decision making is omnipresent. But technology has not augmented human ability to meet these new expectations. There are still many characteristics of human cognition and human interaction with automated systems that form major obstacles to the envisioned optimization of human-automation system performance.

\section{Cognitive Bottlenecks Framework}

The industrial revolution produced machines that led to quantum leaps in human physical work output. While the information age has produced computing machines that could dramatically increase cognitive work output, a corresponding leap has not materialized as expected. We believe this is due to fundamental bottlenecks that exist within human-automation systems. There are multitudes of ways to increase the cognitive work capacity of humancomputer cognitive systems. But first, we must 
understand, from a cognitive perspective, the nature of work, and second, we must understand which aspects of cognitive work have the most room for improvement.

How do you enable one person to do the work of three? First, you identify those places where the information flow between the situation, the human, and the system are most constrained. These places can be thought of as "cognitive bottlenecks." Just as a chain is only as strong as its weakest link, so is the total flow of information and the quality of decision making limited by the most constraining bottleneck. The most constraining cognitive bottlenecks are the ones that most compromise total system performance, and they represent the greatest opportunities for making revolutionary improvements.

Traditionally, human-machine system designers have identified bottlenecks as well-known human limitations such as short-term memory and dual-task performance. This is the standard human factors approach whose stated aim is to design interfaces to machines that mitigate the negative impact of human limitations [31]. This can lead to default function allocation schemes that consider the human and machine component independently when determining which agent is best suited to accomplish some individual task. The fusion of cognitive psychology and information theory resulted in a framework-human information processing - that considers human information-processing bottlenecks in system-oriented terms such as --"input, processing, output" where each stage is limited by the nature of the sub-system that executes it [15]. This approach primarily considers the limitations of the human operator independently of any emergent constraints of a joint human-machine system.

With the rapid proliferation of automation within human-machine systems, researchers now conceptualize information processing stages as potential insertion points for automated aiding. For example, Parasuraman, Sheridan, and Wickens [23] proposed that automation can be applied to four broad classes of functions that correspond to stages of human information processing: Information Acquisition, Information Analysis, Decision and Action Selection, and Action Implementation-acquisition, analysis, decision, and action. This approach requires a priori, static assumptions about the relative utility of automated aiding at each stage without necessarily considering the whole system performance.

However, we prefer a holistic approach that considers the joint human-computer system when identifying bottlenecks to improved system performance. Our rationale is that system designers want to design and develop systems whose joint performance is optimized, and not one in which the human and machine components have been independently optimized. Accordingly, we conducted a holistic analysis of a next-generation human- machine system to identify those cognitive bottlenecks that limited overall system performance. Meta-analysis of past and present research programs identified bottlenecks that have been addressed individually as well as those that have been inadequately addressed. A conceptual framework for designing human-computer cognitive systems is proposed. The Cognitive Bottleneck Framework (CBF) identifies the most constraining areas in a human-computer cognitive system and draws upon research in cognitive psychology, cognitive neuroscience, human-computer interaction design, and computer science.

We identified four primary cognitive bottlenecks that, if not addressed, will negatively impact the quality and tempo of decision making in next generation humancomputer cognitive systems. Generally, the four bottlenecks, within the information processing flow, address sensing and assessing the environment, processing that information, sharing it between the human and the automated information processing systems, and acting upon the environment based on the combined human/automation interpretation of events and identified courses of action. The four cognitive bottlenecks are described below in the order of logical information flow:

Information overload. The human user cannot manage the vast amounts of information afforded by sensor, database, communication, and display advances.

Sequential cognitive processing. While information can be presented to users in parallel, humans are essentially serial processors addressing single threads or tasks at a time. Efficient parallel processing by humans is an exception. Even when possible it due to the efficiency of over-learned tasks (e.g., driving a car and talking on the phone), we become more susceptible to errors.

Narrow user input capabilities. The means of telling the system about their knowledge, questions, and objectives is overly constrained - the system has more sophisticated means of communicating to the human than the human has of communicating to the system.

Function misallocation. The evolutionary development of automation has defined the human user's role and tasks by default rather than by design, leaving them with tasks for which they are cognitively ill-suited.

The overall purpose of CBF approach is to "rightsize" all four of these cognitive bottlenecks, to remove the constraints that restrict information flow, and to better fit the information channels to the abilities of either the human or computer agent.

Furthermore, advances in non-intrusive neurophysiological monitoring techniques provide a vital feedback loop that can inform the automated system about 
the state of its human counterpart. Instead of constraining system design to a "one size fits all," static model of human cognitive limitations, systems can now dynamically adapt the interface to accommodate moment-to-moment changes in an individual operator's cognitive state. This revolutionary feedback mechanism will enable systems to dramatically reduce bottlenecks 1,2 , and 3 , and to a lesser extent 4. For each of the identified bottlenecks we first elaborate on the nature of the problem before discussing the relevant research that supports the conceptual foundation for CBF. Additionally, we discuss some of potential technical approaches to illustrate how the CBF approach can be leveraged for the highest impact on overall joint system cognitive performance. Finally, we describe the implications that neurophysiological feedback has on next generation adaptive systems.

\section{CB1: Information Overload}

Today's systems have the bandwidth and information accessibility to inundate users with information that they cannot effectively acquire and analyze in time to make time critical decisions; tomorrow's systems will be even more informationally powerful. For example, future users will be faced with high-density visual displays that update continuously, as well as incessant voice communication, all while or performing other tasks (e.g. physical like navigating a vehicle or cognitive like developing plans on computer display). This input overload creates bottlenecks at the two early stages of processing information acquisition and analysis, also referred to as extraction and integration - and effectively managing multiple tasks which constrain user's ability to process visual and auditory input in many dimensions; process spatial information from the outside world (can include complexity such as embedded figures, emergent depth information, motion, topography); integrate verbal information (written and spoken language); ignore ambient noise (location, operational state of vehicles); perform quantitative assessment on new information (requiring mental arithmetic); map data and graphical information (charts, forecasts); discern non-verbal information (for example, intent recognition/emotional states of friendly and enemy soldiers); and process additional information (e.g. tactile, kinesthetic cues).

Interactions with complex systems require users to review available information and integrate task-relevant information in working memory in order to have an internal representation of the problem space - one that can be manipulated and analyzed to finally reach some decision [13]. If the user is overloaded with information, they expend precious cognitive resources filtering out irrelevant information which takes additional time and contributes to temporal decay of their representation in working memory [8]; consequently, users are often required to make time-critical decisions based on impoverished mental models of the problem space.

Within these dimensions, a further consideration is the amount of structure in the data. A human's input varies by both the amount of embedded codes and the quality (signal to noise ratio) of the data. Generally, this "raw" data is unformatted and requires further cognitive processing, in contrast to finely tuned "refined" information that can be used much more efficiently. However, unformatted, uncoded data may be tightly bound to the task and so may be the preferred version of the information. For example, a warrior may need to identify a single data point of weather information or the latitude/longitude of an enemy position. Examples include text messages that can vary in syntactic and semantic complexity as well as vocabulary, maps and charts that are full of extraneous irrelevant material that must be actively filtered, tables and charts of varying size and complexity, icons and symbols that need to be interpreted, and video scenes that can vary in resolution and detail. Overall, the inevitable need of the human to filter and reinterpret information contributes to information overload.

Wickens' Multiple Resource Theory [31] describes anticipated perceptual and attentional conflicts for Verbal/Spatial and Visual/Auditory input. In addition to input conflicts, working memory is subject to retroactive interference that is a function of the information codeitems encoded visually interfere most with other visual items [16] whereas verbal items interfere most with other verbal items [29]. Yet another constraint on a user's ability to acquire and integrate information is temporal decay of the elements within working memory [28]. Consequently, as users forage longer for relevant information under information overload conditions, their working memory representation degrades as a function of time.

The aforementioned human cognitive constraints input restrictions, interference of encoded elements, and temporal decay of stored element - are exacerbated by information overload. Therefore, any advanced cognitive system must mitigate the negative impact of information overload without sacrificing information dominance. Advanced information management technologies help humans cope with these inherent limitations, including information filtering and formatting that explicitly support users in integrating disparate information. For example, adaptive filtering, data aggregation, clustering, and advanced visualization have been integrated in complex system in order to mitigate information overload. Depending on the nature of the decision making task, these techniques have proved more or less effective. Given the aforementioned cognitive constraints, techniques that reduce the amount of information integration across time and space have been very effective. 
When compared to more advanced model-based techniques, these approaches are relatively straightforward since they do not necessarily require that the computing platform be "cognitive" or "aware."

\section{CB2: Sequential Cognitive Processing}

Future human operators will be making quick, critical decisions based on unpredictable situations in domains where parameters and tasks are fluid, interdependent, and intricate. The work environment is characterized by real-time information availability and accessibility, expedited decision making for rapid deployment, use of powerful multi-modal workstations, and with fewer human resources available. In short, a single human will be digesting volumes of multivariate data before making quick decisions as the data deluge continues unabated. He or she will not have the luxury of deliberate serial processing; he or she will embrace an information-rich environment in which near real-time data such as mission summaries, tactical data, alerts and advisories, plans, asset management information, and courses of actions flow out of broadband satellite transmission pipes [20]. To avoid drowning in data, their primary challenge will be maintaining situation- and taskawareness in this rapid-fire multitasking environment.

Compact Oxford English Dictionary defines multitasking (MT) as: "the ability to perform concurrent tasks or jobs by interleaving." Multitasking requires more than interleaving: it also includes prioritization, planning, and prospective memory [12]. Burgess [9] foretells the work environment in the Age of Information characterized as a multitasking environment as one with many tasks to be done, differing task characteristics, task interleaving required, self determined adequate performance, one task at a time due to cognitive constraints, no immediate feedback, and delayed intentions as the situation does not always signal when to return to task.

Unfortunately, such a dynamic, data-rich, and fast-paced environment severely challenges the capacity of human cognition. Although humans are capable of performing multiple tasks concurrently, they typically support this by task switching - not doing two separate tasks at once, but rapidly switching between tasks. Adams, Tenney, and Pew [2] concluded that humans can allocate attention to only one task at a time and multiple task management consists of working on one task while "queuing" all others. The sequential cognitive processing bottleneck produces problems when humans attempt to multi-task, such as: operational errors and delays due to inefficiencies in multitasking; tasks or sub-tasks are repeated or omitted; MT performance often is slower than separate-task performance; work overload contributes to non-graceful performance degradation; cognitive tunneling; cognitive fatigue; misdirected attention leads to less important tasks being performed before or instead of more important tasks; and confusions, distractions, disruptions, forgetting, etc., all of which contribute to poor performance.

There are many models of multitasking. Wickens' Multiple Resource Theory [31] accounts for multitasking performance decrements due to interference between tasks demanding similar resources. With multitasking, one can perform two tasks simultaneously with little decrement to performance so long as the tasks are from non-adjacent areas of the cube. Burgess et al.[10] postulated a cognitive neuroscientific model of multitasking functions with three interdependent processes corresponding to neuroanatomically distinct cortical regions.

Another issue is the non-graceful degradation of performance under stress: There are many well documented effects of stress on cognitive performance. One of particular interest in a high workload multitasking environment is the general performance decrement referred to as the "Yerkes-Dodson law" [32] - the fact that the relationship between performance and emotional arousal [stress] can be characterized as an "inverted U," indicating that a moderate arousal level optimizes performance, and low and high arousal levels lead to a performance drop-off. A significant design challenge is that performance degradation associated with high stress is steep (the vertical leg of an inverted $U$ ) and non-graceful. When workload levels are severe, instead of systematic and prioritized off-loading of tasks and goals, humans typically lose the ability to function across the board, and all tasks suffer. This suggests that the multitasking executor shuts down during severe stress.

In summary, there is compelling, converging evidence to the nature, neural location, and limitations of human multitasking. Accordingly, we must consider this as the first and primary cognitive bottleneck in the system. Minimizing this bottleneck requires first identifying those conditions under which humans exhibit superior and inferior multitasking performance so we can design a human-computer interaction system that optimally engages the neuroanatomical and cognitive correlates of multitasking.

\section{CB3: Narrow User Input Capabilities}

This cognitive bottleneck deals with the difficulty the human user has entering information, questions, intentions, and so forth into the system. Part of the reason this bottleneck exists is that the technology and human factors communities have put more emphasis on the problem of getting information from the system to the user than from the user to the system. An analysis of the number of display-related papers (78\% display vs. $17 \%$ controls and 5\% mixed) presented at the Human Factors and Ergonomics Society over the past ten years (1989- 
1998) illustrates this bias [27]. One consequence of this bias is that the bandwidth of the communication channel from the system to the human is much higher than from the human to the system. The system has very sophisticated, flexibly formatted graphic displays with which to transmit information to the human, and the human typically has a keyboard, a cursor control device, and possibly speech recognition with which to transmit information to the system. The information capacities of these various devices are highly unbalanced in favor of the system.

Compounding the psychophysical limitations of current user interfaces are all the other difficulties the human has getting the system to "understand" his or her knowledge of the situation, intentions, questions, and so forth. These include: (1) the system's lack of knowledge of the context, which requires the human to take on the overhead of filling the system in; (2) the need for the human to translate from task logic to system logic; (3) the limited, predefined set of functions that the system typically provides, which requires the human to work around system limitations, select functions that approximately meet their needs because there are none that exactly meet those needs, and interpret the system's behaviors and recommendations with these limitations in mind; and (4) the parallel nature of system output to the human vs. the serial nature of human input to the system.

One of the limiting factors in human-system communication is that the user must fit whatever predefined functions the system provides to meet his or her task needs. This is often straightforward, but there are many cases where the system may not provide the precise function needed by the user, and the user must find a workaround. Workarounds may involve using approximations, which the user must account for when interpreting system outputs, or using provided functions in ways that were unintended by the system designer ("tricking" the system). In any case, workarounds require some cognitive overhead, as the user works to invent a way of bending the system to meet his or her needs.

Research has been done to address some identified psychophysical limitations, by developing systems that respond to gestures, speech, pen input, sketches, and other means. However, the ease of use and the convenience of multi-modal input are often determined by how well the underlying functional logic supports all of these input means. By defining system functional logic to equally support all input means, the user can seamlessly mix and match input means for optimal efficiency and convenience.

\section{CB4: Function Misallocation}

The fourth cognitive bottleneck is related to the allocation of functions between the human and the automated decision-support system. Function allocation involves the distribution of work between humans and automated systems. In 1951, Paul Fitts published a list of the functions best suited to humans or machines [11]. Function allocation decisions have been based on this paradigm ever since: compare humans to computers and assign tasks accordingly. In order to do this comparison, however, all tasks have to be reduced to a common framework, usually mathematical or technology-based [14]. Consequently, function allocation decisions have been driven more by available technology than by user needs, optimal role assignments, or an understanding of the differences between human cognition and computer algorithmic logic. Often the human roles are relegated by default, namely tasks that are too technologically difficult or expensive to automate. What is needed is a flexible, complementary (rather than comparative) approach toward function allocation in the context of both the design and execution stages of human-computer systems.

A variety of guidelines and function allocation processes and rules have been developed. Although, it should be pointed out that "there is no cookie cutter" for performing function allocation [24]. Many decisions are involved in function allocation. The primary one has traditionally been to decide who or what performs a function or task with the traditional choices being (1) function performed by human, and (2) function performed by automation. Bailey [5] describes three types of function allocation strategies: (1) comparison allocation: compare skill requirements and performance criteria between humans and automation for each function; (2) leftover allocation: automate as many functions as technology will permit, and allocate leftover duties to the humans; and (3) economic allocation: where the primary allocation criteria is whether it is more economical to automate or have humans perform a function. Price [24] describes four rules or sets of criteria for allocating functions: (1) mandatory criteria, unambiguously assigning tasks to humans or automation based on the inability of one or the other to perform them, (2) "balance of value" criteria, similar to the comparison method of Bailey [5], which assumes that many functions can be performed by either humans or automation and that the designer should compare performance or efficiency of operation, and allocate based on the balance of value from the comparisons, (3) cost, and (4) "affective or cognitive support," which describes some of the aspects of human performance that today would likely fall under the category of human-centered design issues (e.g. cognitive support refers to the human need to be informed to be prepared to perform actions or make decisions).

Most work in function allocation methods has focused on the "balance of value" or "comparison" rules (e.g., [11][19][24][25]). The fundamental approach has been termed MABA-MABA (Men Are Better At, 
Machines Are Better At) or a "Fitts List" approach. While comparison criteria have dominated thinking about function allocation, it should be noted that advocates of MABA-MABA criteria, including Fitts [11], recognize that allocation should not be made on these criteria alone.

While most of this research focuses on a dichotomous choice, in complex system design there exists a third category of "shared performance." This is illustrated in three-level function allocation taxonomies such as those proposed by Meister [18], McGuire et al. [17], and Tenney, Rogers and Pew [30]. Unfortunately, there has not been a detailed, standard for what "shared" performance means in terms of percentages of a given function performed by humans and automation. The nature of shared human-automation performance requires many other decisions to be made as well. Many function allocation taxonomies have characterized the decisions that must be addressed [19][7][4].

There are three fundamental flaws in function allocation that rely only on a direct comparison approach. First, the comparison of single function or task performance as a basis of allocation does not account for performance and workload effects resulting from the aggregate of tasks assigned to the human. The second flaw is the failure to recognize the repercussions of designing a system that by necessity relies on the human to take over automated functions in the case of automation failure. Reason [26], for example, suggests that human operators are necessary primarily because designers neither foresee all operational situations nor provide automated measures for all contingencies. Humans are ill-suited for passive monitoring [22]. The third is the failure to appreciate the interaction between sub-task execution and management activities such as mission planning, objective setting, and managing emergencies [1][3]. Once again, if one considers only performance of isolated functions and tasks in determining function allocation, many tasks may be automated which are relevant to mission planning and awareness, so users are left uninvolved and uninformed, compromising their ability to perform higher level mission planning tasks.

MABA-MABA criteria, therefore, must be augmented by comparison principles that look at the effects of allocation of individual functions and tasks on human workload, mission management, and performance under stress. Direct comparison of automation and human performance on individual functions and tasks under normal conditions, must not be the sole basis for comparison. Consideration of overall human-computer performance, even at the expense of sub-optimization of local and specific task performance, is a primary tenet of a human-centered design philosophy [21].

\section{Implications for System Design}

The identification of the four cognitive bottlenecks is only the first step in optimizing the throughput of the human-machine collaborative system. Whether it is the exploitation of multiple resource theory to present information in an underutilized modality to minimize information overload or the dynamic allocation of functions based on current human capabilities, potential cognitive bottleneck mediations have to be identified. We will provide an example of how this approach can inform the design of a human-machine system. One such application is the design of an adaptive system that accounts for each of these cognitive bottlenecks and uses the dynamic measurement of the human cognitive state to adjust the automation's level of involvement to maximize the joint human-automation system performance.

By way of example, we provide a brief description of an Augmented Cognition (AugCog) system that is currently the focus of a Defense Advanced Research Projects Agency (DARPA) program. The project objective is to extend the cognitive work capacity of a humancomputer warfighting system by augmenting the human cognitive capabilities. There are four major components to the AugCog system: (1) a cognitive workload assessor which uses neurophysiological and physiological measures to sample the current state of the warfighter's cognitive capabilities, (2) an augmentation manager that makes decisions of how to configure the adaptive system work environment based on its model of the environment, the user, and the tasks, (3) a human-machine interface that is a configurable multi-modal user interface, and (4) the platform which is the application domain, whether it be the flight deck of an aircraft, the cockpit of a ground vehicle, or a dismounted soldier.

The big question is how do we exploit what we know about the cognitive bottlenecks to drive the development of an AugCog system? Several approaches for addressing the four cognitive bottlenecks within a larger biocybernetic adaptive automation system are briefly discussed below to provide examples of how the knowledge of human processing and adaptive automation can be exploited. For each approach we have identified what bottleneck(s) have been mitigated.

Neuroergonomic adaptive automation. A system that can unobtrusively measure an operator's cognitive capacity has the potential to relieve each of the four identified bottlenecks. Neurophysiological measures that indicate the operator is cognitively overloaded can inform the automation (CB3: Input) that the human processing required (CB2: Sequential Cognitive Processing) to manage the incoming information (CB1: Information Overload) is reaching its limit and the system should offload some tasks to the automation (CB4: Function 
Allocation). This biocybernetic feedback loop can optimize the human and machine's input to the system.

Adjustable Autonomy. This approach calls for a moving middle ground of human-automation interaction: one that can assume more or fewer of the duties depending on the situation and the level at which the user wants or is able to interact with the system (CB4)).

Mitigating cognitive weaknesses. Ideally, function allocation would relieve humans of the responsibility for tasks for which they are not well suited (CB4), thus freeing them up to focus on those tasks for which humans are uniquely well suited (e.g., insight, inquisitiveness, creativity, exercise of authority).

Information Modality Management. Wickens' Multiple Resource Theory contends that the dynamic allocation of incoming information to the most readily available attentional resource pool will avoid overtaxing the operator (CB1).

Multi-tasking support. In order to reduce the time required to resume suspended tasks, an automated system needs to retain memory of the task status, update the operator on any changes to the task status that have occurred during task suspension, and remind the operator to return to the suspended task when appropriate (CB2).

\section{Abstraction of Information Presentation} Sometimes users are overloaded not because of the volume of information, but rather because the available information is not at the appropriate level for a given task, their expertise, or their current role. A system that could store, reason, and present information at different levels of abstraction to support different users doing disparate tasks would mitigate information overload (CB1).

Cross-modality functional logic. If a single, consistent set of functional logic is defined that has equal counterparts in all modalities, the user can mix and match input methods to accommodate his or her current task demands. Because all input methods comply with the same underlying functional logic, the user is not constrained to finish an operation the way they started it, or to use a particular input method that may be difficult in the current mix of tasks (CB3).

\section{Conclusions}

The Cognitive Bottleneck Framework has been presented as a way of identifying the most constraining aspects of human-automation system design. A particularly challenging type of human-machine system is an adaptive system, where the automation proactively changes the work environment to increase joint system performance. In such a system, the information flow between human and automation are particularly subject to the bottlenecks identified in CBF, severely compromising overall joint system performance. By way of example, several technical approaches were presented to mitigate the cognitive bottlenecks identified for an Augmented Cognition System, where neurophysiological feedback is utilized to close the loop in a highly adaptive system, with the ultimate goal of multiplying the cognitive work a human can perform. The CBF allows system designers to identify the areas that need the most attention, and assess approaches to focus design activity where it will have the most impact on overall joint human-automation system performance. The overall purpose of CBF-based approach to design is to "right-size" all four cognitive bottlenecks, to remove the constraints that restrict information flow, and to better fit the information channels to the abilities of the human.

\section{Acknowledgments}

The authors would like to thank Dr. Christopher Miller, Steve Metz, Dr. Victor Riley, and Danni Bayn for work that directly contributed to the development of the CBF framework. The authors would also like to thank Murray Cooper for support and sponsorship.

\section{References}

[1] Abbott, T. (1993). Functional categories for future flight deck designs. NASA Technical Memorandum TM109005. Hampton, VA: NASA Langley Research Center.

[2] Adams, M. J., Tenney, Y. J., and Pew, R. W. (1995). Situation awareness and the cognitive management of complex systems. Human Factors, 37(1), 85-104.

[3] Alter, K. W., Erickson, J. B., Goins, R. T., Hofer, E. F. Koehn, W. L., Miles, W. L., Mowry, R. S., and Pfaff, T. A. (1995). High speed research flight deck design and integration flight deck concepts. Seattle, WA: Boeing/McDonnell Douglas Industry Team.

[4] Alter, K. W., and Regal, D. M. (1992). Definition of the 2005 flight deck environment, (NASA Contractor Report 4479), Seattle, WA: Boeing Flight Deck Research.

[5] Bailey, R. W. (1982). Human Performance Engineering: A Guide for System Designers. Englewood Cliffs, NJ: Prentice-Hall.

[6] Baker, M. P., and Wickens, C. D. (1995) Human Factors in Virtual Environments for the Visual Analysis of Scientific Data. NCSA-TRO32 and Institute of Aviation ARL-95-8/PNL-95-2. 

2003 IEEE International Conference on Systems, Man, and Cybernetics. Washington DC, October 5-8.

[7] Billings, C.E. (1996). Aviation automation: The search for a human-centered approach. Hillsdale, NJ: Lawrence Erlbaum Associates)

[8] Baddeley, A. (1986). Working Memory. Oxford: Clarendon Press.

[9] Burgess PW, (2000), Strategy application disorder: the role of the frontal lobes in human multitasking", Psychological Research, 63(3-4), pp 279-288.

[10] Burgess, P.W., Veitch, E., de Lacy Costello, A., and Shallice, T. (2000). The cognitive and neuroanatomical correlates of multitasking. Neuropsychologia, 38, 848863.

[11] Duncan, J. (1986). Disorganisation of behaviour after frontal lobe damage. Cognitive Neuropychology, 3, 271-90.

[12] Ellis, J. (1996). Prospective memory or the realization of delayed intentions: A conceptual framework for research (pp. 1-22). In M. Brandimonte, G. Einstein, and M. McDaniel, (Eds.) Prospective Memory: theory and applications.

[13] Gentner, D. and Franks, J. J. (1983). Mental Models. Hillsdale, NJ: Lawrence Erlbaum.

[14] Jordan, N. (1963). Allocation of functions between man and machines in automated systems. Journal of Applied Psychology, 47, 161-165.

[15] Lindsay, P.H. and Norman, D.A. (1977). Human Information Processing: An Introduction to Psychology, Second Edition. Academic Press, New York.

[16] Logie, R., Zucco, G. M. \& Baddeley, A. D. (1990) Interference with visual short-term memory. Acta Psychologica, 75, 55-74.

[17] McGuire, J. C., Zich, J. A., Goins, R. T., Erickson, J. B., Dwyer, J. P., Cody, W. J., and Rouse, W. B. (1991). An Exploration of Function Analysis and Function Allocation in the Commercial Flight Domain. (NASA CR4374). Long Beach, CA: McDonnell Douglas Aircraft Company.

[18] Meister, D. (1985). Behavioral Analysis and Measurement Methods. New York: Wiley Interscience.

[19] Mertes, F. and Jenney, L. (1974). Automation applications in an advanced air traffic management system: Vol. Ill, Methodology for man-machine task allocation (Report No. DOT-TSC-OST-74-14-111). McLean, VA: TRW, Inc.
[20] Morrison, J., Kaufmann, K., Dong, H., and Fehér, B. (2001). Global 2000- Prototype Knowledge Wall (SPAWAR presentation). Available online at: http://wwwtadmus.nosc.mil/kwintro_files/frame.htm\#slide0073.htm.

[21] Norman, D.A., and Shallice, T. (1986). Attention to action: Willed and automatic control of behaviour. In R. Davidson, G. Schwartz, and D. Shapiro (Eds.) Consciousness and self regulation (vol 4).

[22] Parasuraman, R. and Bowers, J.C. (1987). Attention and vigilance in human-computer interaction. In A. Gale and B. Christie (Eds.) Psychophysiology of the Electronic Workplace. (pp. 163-194). London: Wiley.

[23] Parasuraman, R., Sheridan, T.B., and Wickens, C.D. (2000). A model for types and levels of human interaction with automation. IEEE Transaction on Systems, Man, and Cybernetics--Part A: Systems and Humans, 30(3), 286297.

[24] Price, H. (1985). The allocation of functions in systems. Human Factors, 27(1), 33-45.

[25] Price, H. E., Maisano, R. E., and Van Cott, H. P. (1982). The allocation of functions in man-machine systems: a perspective and literature review. (NUREG/CR-2623). Oak Ridge, TN: Oak Ridge National Laboratories.

[26] Reason, J. (1990). Human error. New York: Cambridge University Press.

[27] Riley, V. (2000). Personal communication.

[28] Rubin D.C., Hinton S. and Wenzel A (1999) The precise time course of retention, Journal of Experimental Psychology, 25, 1161-1176.

[29] Shiffrin, R. M. (1973). Information persistence in short-term memory. Journal of Experimental Psychology, $100,39-49$.

[30] Tenney, Y. J., Rogers, W. H., and Pew, R. W. (1995). Pilot opinions on high level flight deck automation issues: Toward the development of a design philosophy. (NASA Contractor Report 4669). Hampton, VA: NASA Langley Research Center.

[31] Wickens, C.D., (1992). Engineering psychology and human performance (2nd ed). New York: Harper Collins.

[32] Yerkes, R.M. and Dodson, J.D. (1908). The Relationship of Strength of Stimulus to Rapidity of Habit Formation. Journal of Comparative Neurology and Psychology, 18, 459-482. 